\title{
Design and Evaluation Method of Wireless Fire Detection Node Based on Multi-source Sensor Data Fusion
}

\author{
Huang Ye ${ }^{1,2}$, Wang Xiaogang ${ }^{1,2, *}$, Gan Shuchuan ${ }^{1,2}$ \\ ${ }^{1}$ School of Automation \& Information Engineering, Sichuan University of Science \& Engineering, Yibin, China \\ ${ }^{2}$ Artificial Intelligence Key Laboratory of Sichuan Province, Sichuan University of Science \& Engineering, Yibin, China
}

Email address:

wxg_zf@163.com (Wang Xiaogang)

${ }^{*}$ Corresponding author

\section{To cite this article:}

Huang Ye, Wang Xiaogang, Gan Shuchuan. Design and Evaluation Method of Wireless Fire Detection Node Based on Multi-source Sensor Data Fusion. International Journal of Sensors and Sensor Networks. Vol. 9, No. 1, 2021, pp. 19-24. doi: 10.11648/j.ijssn.20210901.13

Received: January 17, 2021; Accepted: January 25, 2021; Published: January 30, 2021

\begin{abstract}
In view of the problem that the traditional fire detection node is not intelligent enough for data processing when building fire occurs, a design and evaluation method of wireless fire detection node based on multi-source sensor data fusion is proposed. Firstly, the temperature and humidity sensor, carbon monoxide sensor and smoke sensor are selected to collect three kinds of fire information eigenvalues at the same time. Secondly, the analysis and processing method of two-level data fusion is established, and the fire information is processed in STC89C52 microcontroller. The first level data fusion uses fuzzy proximity algorithm to get the eigenvectors of environmental parameters, so that the measured values are closer. The second level data fusion uses D-S evidence theory to analyze the data systematically. According to the probability distribution function after fusion, more accurate decision results are obtained. Finally, the fire signal is sent to the superior data management center through ZigBee wireless transmission network, and the alarm signal is triggered in time. The whole node design gives a complete hardware selection and software data fusion processing method. Compared with the traditional fire detection node, it significantly reduces the false alarm rate. At the same time, the node has good application effect in the experiment, and realizes a wireless intelligent fire detection node with low cost, wide application range and high intelligence.
\end{abstract}

Keywords: Fire Detection, Two Level Data Fusion, Fuzzy Proximity Algorithm, D-S Evidence Theory

\section{Introduction}

Fire has always been one of the serious natural disasters in our country. If there are no effective fire monitoring measures in the fire area, it will lead to immeasurable economic losses and casualties. Especially in modern times, with the continuous development of urbanization, the fire hazards in all kinds of buildings have been strengthened, which has always been a major threat to the safety of people's lives and property, It is the key research object of all kinds of fire protection system to detect and extinguish the fire information timely and accurately in the initial stage of fire. On the other hand, with the rapid development of modern science and technology, the design of more convenient, fast, high accuracy wireless intelligent fire detection node has gradually become the key research content.

At present, many fire detection nodes in the market simply judge whether there is a fire according to whether a single fire feature exceeds the threshold. This way of data processing can't analyze the data scientifically and effectively, which often leads to the failure to accurately judge whether a fire occurs. In view of the above phenomenon, this paper designs a wireless intelligent fire detection system based on two-level data fusion. The node can collect multiple fire signs at the same time, and combine two data fusion algorithms to analyze and judge the fire features step by step. The purpose is to effectively improve the accuracy of fire detection and reduce the false alarm rate $[1,2]$. The wireless intelligent fire detection node consists of four parts: fire information acquisition unit, data fusion processing unit, wireless data transmission unit and power supply unit. Because the fire signal detection is more complex than other typical signal detection, and the monitoring range is wide, we can network based on ZigBee technology, realize the design of wireless transmission module, and reasonably layout the system 
structure, and can carry out corresponding data fusion for different types of fire parameters, so as to get a more accurate method to judge the fire, On the other hand, ZigBee technology has low power consumption, low wiring cost and simple maintenance, which is suitable for building a wide range of wireless fire detection node network [3]. The design of wireless intelligent fire detection node consists of the following parts:

\section{(1). Design of Fire Information Acquisition Unit}

The fire information acquisition unit includes three types of sensors, namely temperature and humidity sensor, carbon monoxide sensor and smoke sensor. Its principle is to collect data in real time by deploying different types of sensors in many places to form multiple autonomous nodes. These nodes can be combined with wireless transmission module to form an integrated network structure, It has the function of real-time collection and timely feedback, which is convenient for the central controller to process the relevant data and parameters.

(2). Design of MCU Data Fusion Processing Unit

MCU data fusion processing unit is the core of the whole fire detection node, which is responsible for the normal operation of the whole node. Its main function is to use intelligent algorithm to analyze and process all kinds of data collected, get more accurate decisions, and effectively manage the realization of each task of the node [4].

\section{(3). Design of Wireless Data Transmission Unit}

The data wireless transmission unit uses ZigBee technology for the establishment and networking of the sensor network. After collecting the fire information detected by the fire information acquisition unit, it carries out relevant data transmission. Users can also directly or indirectly control the network through computers, mobile phones and other terminals [5].

The overall structure of fire detection node is shown in Figure 1 .

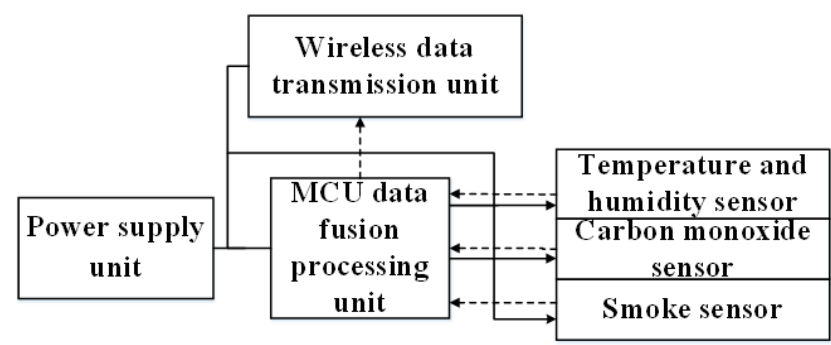

Figure 1. Overall structure.

\section{Design of Fire Information Acquisition Unit}

In the process of fire, it is usually accompanied by rapid changes in temperature and humidity, and the concentration of carbon monoxide and smoke increases rapidly. Therefore, it is not possible to detect a single fire parameter to determine whether a fire occurs or not, so as to avoid the occurrence of high false alarm rate. The design of detection node should be based on the collection of these three information [6], The fire information acquisition unit is mainly composed of Am2322 digital temperature and humidity sensor, Tgs5141 carbon monoxide sensor and NIS-09C ion smoke sensor.

(1). Am2322b Digital Temperature and Humidity Sensor

Am2322b sensor is a kind of composite sensor with high efficiency and practical temperature and humidity acquisition technology. Its advantages are high reliability and strong stability. In this kind of sensor, it includes high-precision integrated temperature measuring element, capacitive humidity sensor and a microprocessor to process the sensed data in time, which has low power consumption, small size and strong anti-interference ability.

Communication mode of Am2322b sensor is divided into two types, namely single bus communication mode and $\mathrm{I}^{2} \mathrm{C}$ communication mode. When using single bus interface, the system integration can be simplified and convenient, and the signal transmission distance can be about 20 meters, which can meet the needs of various demanding occasions. Users choose the $\mathrm{I}^{2} \mathrm{C}$ communication mode, they can also directly hang on the communication bus without additional wiring. Users of the two communication modes can switch freely according to the actual needs, which has wide application fields and strong practicability. The main technical parameters of Am2322 digital temperature and humidity sensor are shown in Table 1.

Table 1. Parameters of Am2322b digital temperature and humidity sensor.

\begin{tabular}{ll}
\hline $\begin{array}{l}\text { Brief introduction of } \\
\text { parameters }\end{array}$ & $\begin{array}{l}\text { Am2322b digital temperature and } \\
\text { humidity sensor }\end{array}$ \\
\hline Supply voltage & $3.1 \mathrm{~V} \sim 5.5 \mathrm{~V}$ \\
Temperature range & $-40 \sim 80^{\circ} \mathrm{C}$, error $\pm 0.3^{\circ} \mathrm{C}$ \\
Humidity range & $0 \sim 99.9 \% \mathrm{RH}$ error $\pm 2 \% \mathrm{RH}$ \\
Maximum power consumption & $250 \mu \mathrm{A}$ \\
Minimum sampling period & $2 \mathrm{~S}$ \\
\hline
\end{tabular}

(2). Tgs5141 Carbon Monoxide Sensor

Tgs5141 sensor is a kind of electrochemical sensor which can be driven by battery. Its characteristic is that the water reservoir is replaced by a special electrode and its appearance is light. Each sensor has its own bar code, which can be used to identify and print the data obtained by each sensor. While tracking the sensor, it can also save the trouble of using expensive gas calibration procedures and greatly reduce the use cost. Moreover, this carbon monoxide detector has strong anti-interference ability and high sensitivity, It is suitable for the detection of various fire situations. The main technical parameters of Tgs5141 carbon monoxide sensor are shown in Table 2 .

Table 2. Parameters of Tgs5141 carbon monoxide sensor

\begin{tabular}{ll}
\hline Brief introduction of parameters & Tgs5141 carbon monoxide sensor \\
\hline Gas detection & carbon monoxide \\
Detection range & $0 \sim 5000 \mathrm{ppm}$ \\
Temperature range & $-10^{\circ} \mathrm{C} \sim+50^{\circ} \mathrm{C}$ \\
Humidity range & $10 \sim 95 \% \mathrm{RH}$ \\
response time & Within 60 seconds \\
\hline
\end{tabular}




\section{(3). Nis-09c ion Smoke Sensor}

Ion type smoke sensor is a kind of smoke sensor with advanced working principle and superior performance. It has been widely used in various fire alarm systems. Compared with the traditional gas sensitive resistance fire alarm, its performance has improved a lot.

The sensor Nis-09c used in this paper is a kind of sensor which can sensitively detect smoke and dust in the event of fire. Its characteristics are that the sensor contains trace radioactive elements, but it does not affect our use safety. Secondly, the power consumption in the process of use is very low, and the ordinary battery can continue to run for several years. Compared with the photoelectric smoke sensor, its structure is simpler Single, the use cost is also much lower, and the durability has been improved, the output is independent of the smoke color. The main technical parameters of NIS-09C ion smoke sensor are shown in Table 3.

Table 3. Parameters of Nis-09c ion smoke sensor

\begin{tabular}{ll}
\hline Brief introduction of parameters & NIS-09C ion smoke sensor \\
\hline Supply voltage & $\mathrm{DC} 9 \mathrm{~V}$ \\
Temperature range & $0 \sim 50^{\circ} \mathrm{C}$ \\
Humidity range & Less than $95 \% \mathrm{RH}$ \\
Current consumption & $27+/-3 \mathrm{pA}$ \\
Smoke sensitivity & $0.6+/-0.1 \mathrm{~V}$ \\
\hline
\end{tabular}

\section{Design of Wireless Data Transmission Unit}

ZigBee technology is a two-way wireless communication technology with low transmission rate, low power consumption, low maintenance cost and strong anti-interference ability. The three nodes in ZigBee wireless transmission network are coordinator, router and terminal respectively. The main control chip of all nodes adopts CC2530 chip of TI company. CC2530 has excellent RF transceiver and $8051 \mathrm{CPU}$, which has strong practicability and low power consumption It is also low cost and easy to develop. It is suitable for the development of wireless communication technology in various environments. Coordinator plays the role of establishing new network and network maintenance in ZigBee network, and it is the central hub of the whole network Router can act as parent node or child node in the whole ZigBee network, and can realize information forwarding and assist coordinator to maintain the network together. Terminal can only join ZigBee network as a child node device, and communicate with parent node after collecting relevant data Secondly, ZigBee network topology can be divided into star network, tree network and mesh network [7, 8].

When the terminal node of ZigBee wireless transmission network is powered on and initialized, if a nearby network is found, it will send a network access application. After successful network access, it will send a binding request to its parent node. If the binding response is received, it indicates that the binding is successful. At this time, the terminal node can transmit data information to the parent node. If the parent node is a router, it will also forward the information to the coordinator After each node uses ZigBee wireless transmission technology to realize networking, when a node detects a fire, it can use ZigBee wireless transmission network to send the perceived fire signal to the superior data management center and trigger the alarm signal. The terminal node data transmission flow chart is shown in Figure $2[9,10]$.

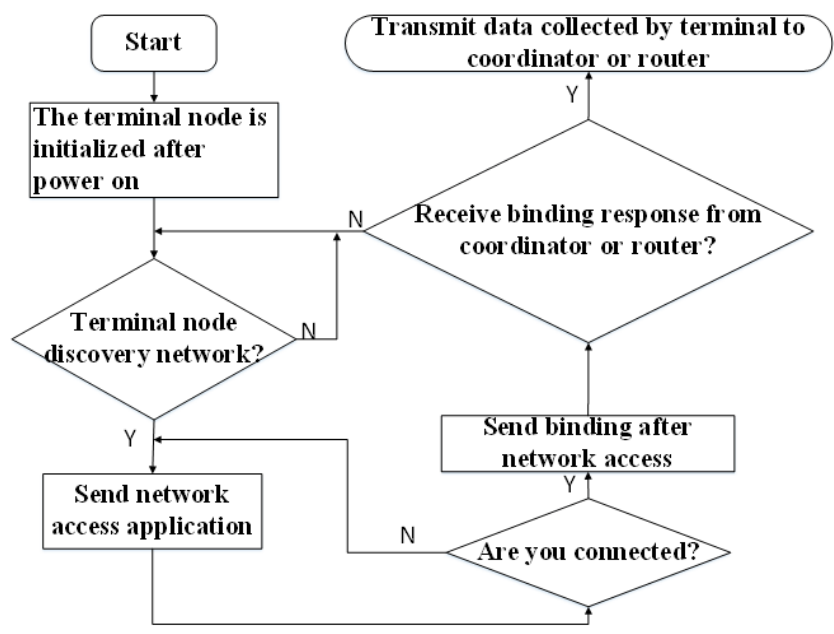

Figure 2. Data transmission process of terminal node.

\section{Design of MCU Data Fusion Processing Unit}

\subsection{Design of Fire Detection Node Circuit}

STC89C52 microcontroller is used as the main control chip in the data fusion processing unit. It is an 8-bit microcontroller with low power consumption and excellent performance. Its main characteristics are that it has $8 \mathrm{~K}$ bytes of program storage space and 512 bytes of data storage space. Meanwhile, it has built-in $4 \mathrm{~K}$ bytes of EEPROM storage space. It can directly use the serial port to download data. It has rich and flexible functions, and is easy to expand and suitable for application Used in various control application systems $[11,12]$.

In the design process, Am2322 digital temperature and humidity sensor, Tgs5141 carbon monoxide sensor and Nis-09c ionic smoke sensor are used in each module. In the design process, the key reset circuit is used. When the reset key is pressed, the rst pin of MCU will appear high level immediately, and then reset. In addition, the internal clock circuit mode of $11.0592 \mathrm{Mhz}$ crystal oscillator is selected. The capacitor connected at both ends of the crystal oscillator is convenient for fast starting vibration and improving stable frequency; The chip ADC0832 with 8-bit split rate and dual channel A / D conversion is used between the smoke sensor module and the single chip microcomputer. In the process of signal conversion, the enable end should be set at low level until the conversion is completed. As shown in Figure 4, the power supply unit includes LM7905 and L78M05 three terminal fixed positive regulators. The power supply unit is connected with each sensor module and MCU microcontroller 
to provide power for the node to collect information and analyze and process data in real time. The overall system circuit diagram and power circuit diagram of the whole node are shown in Figure 3 and Figure 4 below.

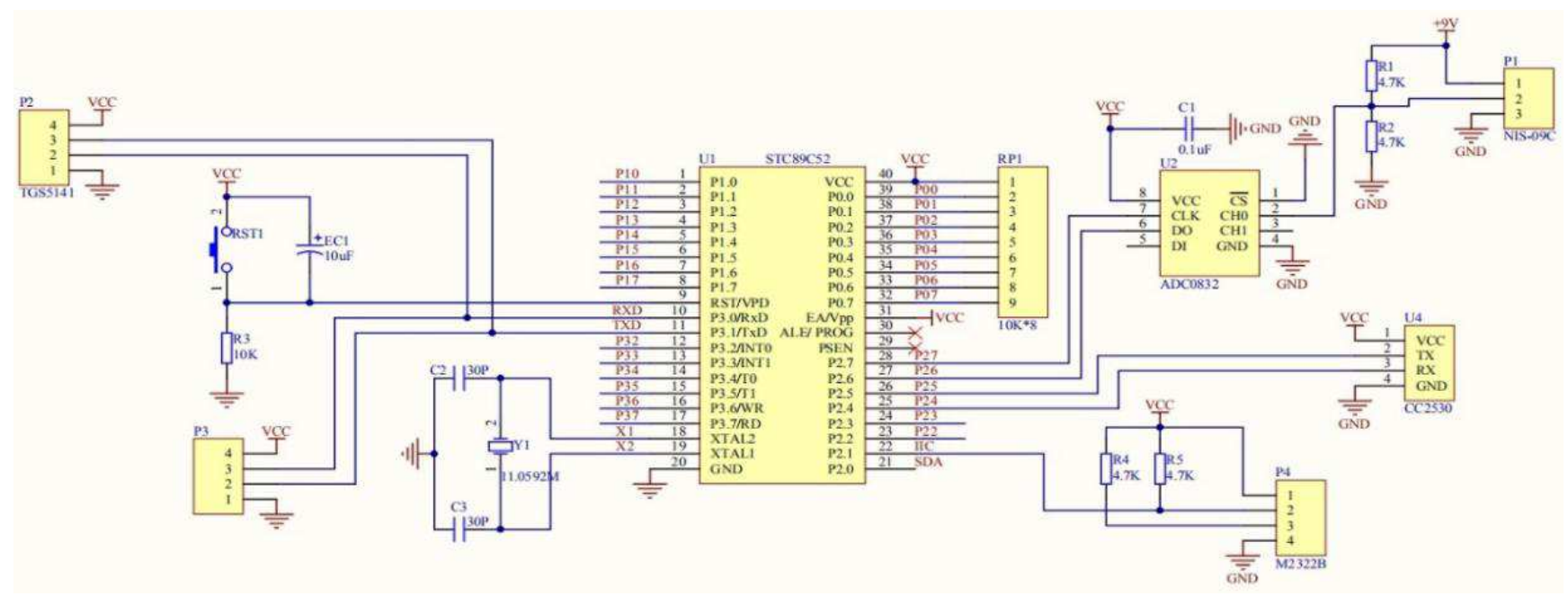

Figure 3. Overall system circuit.

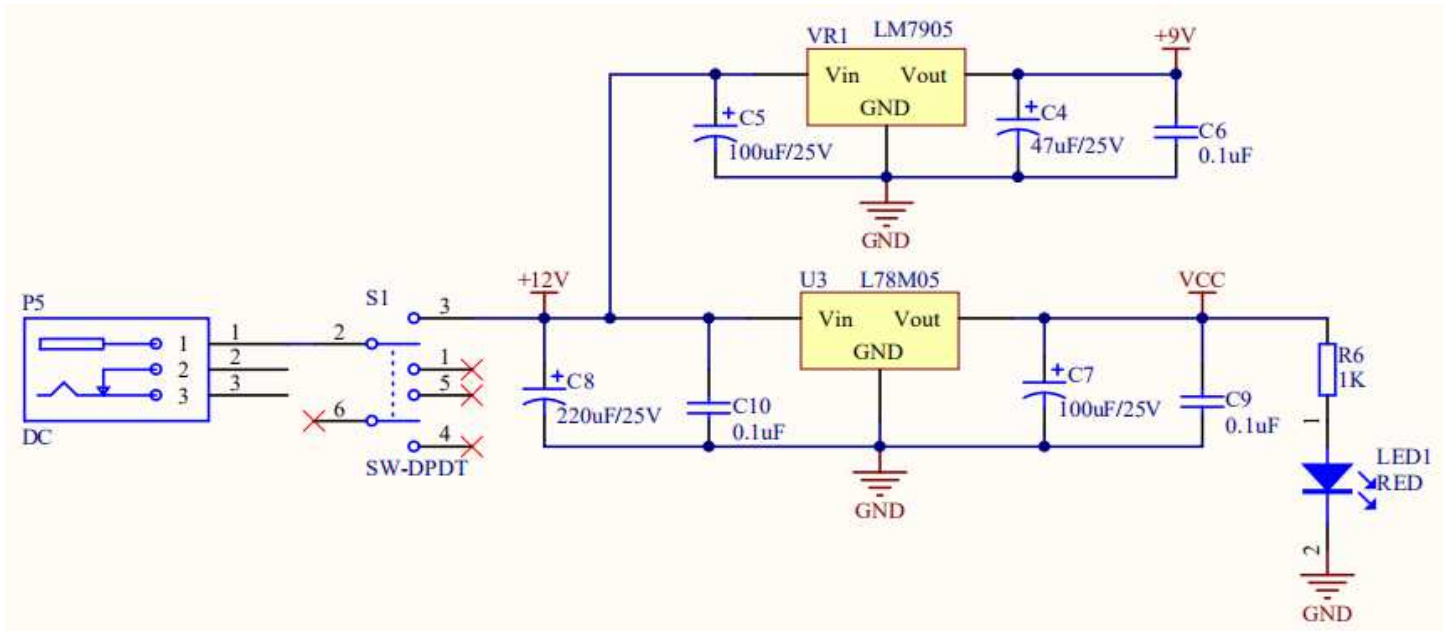

Figure 4. Power circuit

\subsection{Two Level Data Fusion Algorithm}

The algorithm of fire information processing is the key point of the whole fire detection node design. Now in the market, more threshold comparison method is used to judge whether a fire occurs or not, that is, a standard range of temperature and carbon monoxide concentration when a fire occurs is obtained by experiment, a threshold is set in advance, and the collected data value is compared with the threshold value. If it exceeds the threshold value, it can be used This paper concludes that there is a fire, but this traditional judgment method does not have a more intelligent analysis of the data, and the false alarm rate is also high.

Therefore, the information processing method designed in this paper is to process the temperature, humidity, carbon monoxide and smoke concentration data that exceed the threshold by median average filtering method. The principle is to continuously sample $\mathrm{n}$ data of the sample, remove one maximum and one minimum, and then calculate the arithmetic average of the remaining $n-2$ data, in order to reduce the bias caused by interference The first level of data fusion is to fuse the original data collected by the sensor with fuzzy proximity algorithm, so that the data feature vector is closer to the real value, and then the parameters obtained from the first level of data fusion are used as the input, and the second fusion is carried out in D-S evidence theory, so as to get the judgment of whether the fire really happened result. The process of filtering and data fusion is shown in Figure 5.

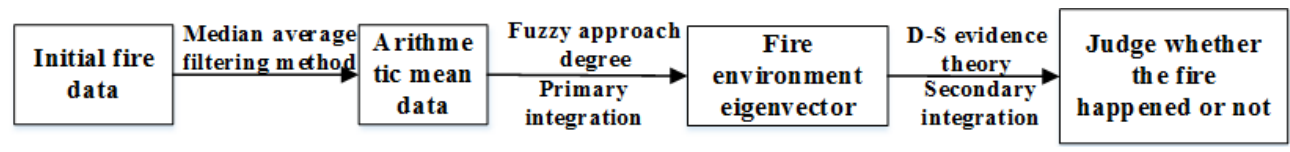

Figure 5. Data fusion process. 


\subsubsection{First Level Data Fusion Processing}

In case of fire, the complex and harsh environment has a certain impact on the process of sensor data acquisition, which will make the deviation. Therefore, in order to further reduce the data deviation, the fuzzy proximity algorithm can be used to fuse the initial data collected when the fire occurs. The specific implementation steps are as follows.

The deviation value of data collected by the same kind of sensor is $x_{i}, i=1,2,3, \ldots n$, In which $i$ Denotes the $i$ Sensors, $n$ means there are a total of sensors of this type, Calculate the average value $\bar{x}$ of all the data collected by the $n$ sensors, we can get the fuzzy closeness degree $y_{i}$ between these data and the mean value $\bar{x}$, which represents the closeness degree between fuzzy sets.

$$
\begin{gathered}
\bar{x}=\frac{1}{\mathrm{n}} \sum_{\mathrm{i}=1}^{\mathrm{n}} \bar{x}_{\mathrm{i}} \\
y_{i}=1 /\left[\left(1+\left|x_{i}-\bar{x}\right|\right)\right]
\end{gathered}
$$

Then the fuzzy closeness degree $\bar{x}$ is substituted into formula (3) to obtain the relative weight $\omega_{i}$. Finally, the primary data fusion result $Z$ collected by the sensor is obtained from formula (4).

$$
\begin{gathered}
\omega_{i}=y_{i} / \sum_{i=1}^{n} y_{i} \\
z=\sum_{i=1}^{n} \omega_{i} x_{i}
\end{gathered}
$$

Then repeat the above steps to fuse the collected temperature, carbon monoxide concentration and smoke concentration data at the first level, and then form the feature vector with the fusion result value to make the data closer to the real value, so as to prepare for the next level of data fusion [13].

\subsubsection{Secondary Data Fusion Processing}

In this paper, the evidence source of D-S evidence theory comes from the results of primary fusion processing of data collected by different types of sensors. According to these different types of evidence sources, the basic probability allocation function $m(x)$ can be obtained, and the function value of each function $m(x)$ is within $[0,1]$. Then the corresponding trust function $\operatorname{bel}(x)$ and likelihood function $p l(x)$ are calculated according to the function $m(x)$, More accurate decision results can be obtained under the prescribed criteria [14].

Where, $U$ is the identification box, in which we can divide the fire into open fire, smoldering fire and no fire. $m(A)$ represents the support for event $A, \operatorname{Bel}(A)$ represents the degree of trust when event $A$ is true, $\operatorname{Pl}(A)$ represents the degree of trust when event $A$ is not false. The decision process is implemented in the identification box $U$, and the subset set is $2^{U}$, and the result can be mapped in the interval [0, 1], In addition, $\varnothing$ is an empty set, and $C$ is the probability result after fusion. The specific steps are as follows:

$$
\begin{gathered}
\sum_{A \subset U} m(A)=1 \\
\operatorname{Bel}(A)=\sum_{B \subset A} m(B)(\forall A \subset U) \\
P l(A)=1-\operatorname{Bel}(\bar{A})=\sum m(B)(\forall A \subset U)
\end{gathered}
$$

Then according to formula (9), the probability distribution function $m(C)$ after data fusion is obtained.

$$
\begin{gathered}
K=\sum_{A_{i} \cap B_{j}=\alpha} m_{1}\left(A_{i}\right) m_{2}\left(B_{j}\right)<1 \\
m(C)=\left\{\begin{array}{c}
\frac{\sum_{A_{i} \cap B_{i}=C} m_{1}\left(A_{i}\right) m_{2}\left(B_{j}\right)}{1-K} \\
0, C=\phi
\end{array}\right. \\
\forall C \subset U, C \neq \phi
\end{gathered}
$$

Where, $m_{1}$ and $m_{2}$ represent the probability distribution function in the recognition fram $U$, and $A_{1}, A_{2} \cdots A_{m}, B_{1}, B_{2}, \cdots B_{m}$ is the corresponding focal element. $K$ is the conflict factor, which can judge the degree of conflict among evidences. When $K$ value approaches 1 , it means that this method is not applicable $[15,10]$.

Finally, according to the fused probability distribution function $m(C)$, more accurate decision results can be obtained, and the information processing flow chart is shown in Figure 6.

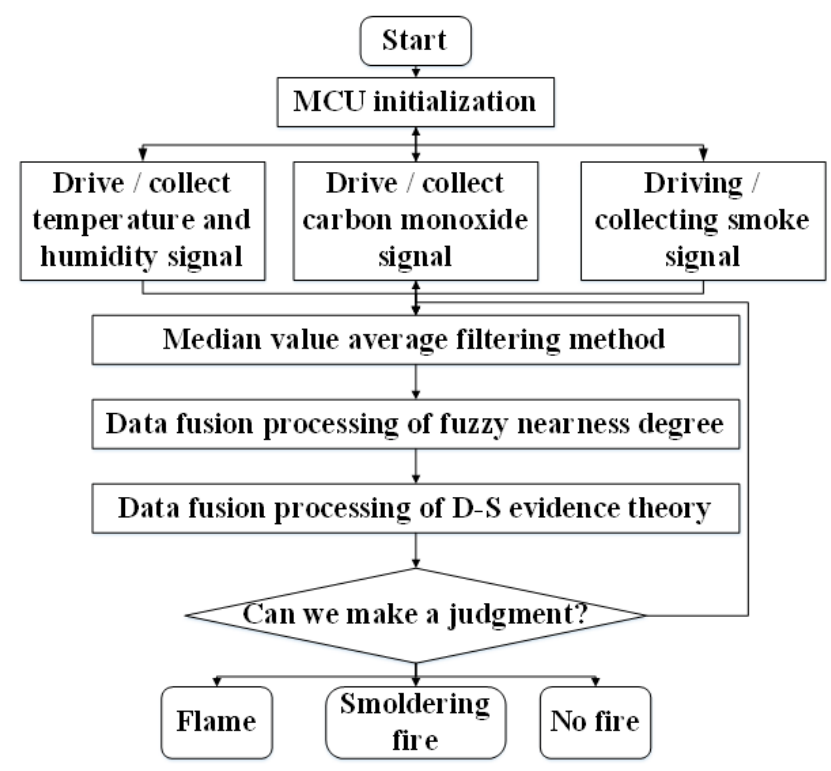

Figure 6. Information processing flow.

\section{Conclusion}

In this paper, the whole fire detection node from the perspective of low development cost, strong practicability, and more optimized system scheduling, based on IAR for 8051 development environment, ZigBee software stack and CC2530 wireless transceiver module, using the above design ideas, a wireless intelligent fire detection node based on two-level data fusion method is designed. The fire information acquisition unit collects the concentration data of temperature and humidity, carbon monoxide and smoke, and sends it to the MCU in charge of data fusion processing unit, and uses the corresponding two-level data fusion Finally, the wireless data transmission unit sends the perceived fire signal to the superior data management center, and triggers the alarm signal 
in time. The node is well applied in the experiment, which greatly improves the accurate performance of the fire detection node. Moreover, the fire detection node has low maintenance cost, obvious performance advantages, and can be widely used It is widely used in building construction.

\section{Acknowledgements}

This work was supported in part by National Natural Science Foundation of China (grant no. 61902268), Sichuan Science and Technology Program (grant no. 2018JY0197, 19ZDZX0037, 2019YFSY0045, 20ZDYF0919, 21ZDYF4052, 2020YFH0124), Foundation of Deyang Open School-City Cooperative Technology Research and Development (Grant No. 2018CKJSD017). Zigong Key Science and Technology Project of China (2020YGJC01).

\section{References}

[1] Luo Xiaoquan, pan Shanliang, Fire alarm system based on multi-sensor data fusion [J]. Data communication, 2019 (06): $22-26,31$

[2] Xufeng Wei, Yahui Wang and Yanliang Dong, "Design of fire detection system in buildings based on wireless multimedia sensor networks," Proceeding of the 11th World Congress on Intelligent Control and Automation, Shenyang, 2014, pp. 3008-3012.

[3] Zhang Bohu, Chen Jianli. Design of fire detection system based on artificial intelligence technology and ZigBee [J]. Fire science and technology, 2008 (01): 49-51

[4] Ning Du, Zhi Long Liu, Huai Guo Dong. Design of Wireless Sensor Network for Fire Detection [J]. 2014, 3265: 657-660.

[5] S. Liu, D. Tu and Y. Zhang, "Multiparameter fire detection based on wireless sensor network," 2009 IEEE International Conference on Intelligent Computing and Intelligent Systems, Shanghai, 2009, pp. 203-206.

[6] Zeng Sitong, Tong Xiaowei, Shen peihui. Design of fire detection system based on wireless multi-sensor information fusion [J]. Journal of Hubei Institute of technology, 2019, 35 (06): 23-27, 32.

[7] Liu Yan, "The reasearch to the signal transmission for fire detector," 2011 International Conference on Electric Information and Control Engineering, Wuhan, 2011, pp. 855-858.

[8] He-zhi Guo, Jun-ling Gao and Ben-yu Huang, "Apply of coal-bunker fire warning system based on ZigBee," 2014 IEEE Workshop on Electronics, Computer and Applications, Ottawa, ON, 2014, pp. 376-378.

[9] Ding min. design of wireless intelligent automatic fire alarm system [J]. New technology and new products in China, 2019 (14): $143-144$
[10] Cheng Wenbin, Du Lei, Liu Yiyi. Design of home fire detection system based on wireless multi-sensor data fusion [J]. Telecom Science, 2017, 33 (09): 174-181

[11] Chen Quan. Design of intelligent fire alarm based on $51 \mathrm{MCU}$ [J]. Electronic production, 2017 (23): 25-28

[12] Zhang Qiang, Zhang Chaolong, Li Nan, Jiang Shanhe, Li Yanmei. Design of intelligent fire alarm based on MCU [J]. Computer knowledge and technology, 2019, 15 (12): 210-212.

[13] Wu Jinghong, Wu Jinghong, Liu Xun. Design of coal mine safety monitoring system based on ZigBee technology and information fusion [J]. Coal engineering, 2017, 49 (10): 55-58, 62

[14] S. Tian and M. Kou, "Fire Safety Assessment of an Underground Parking Area Based on D-S Evidence Theory," 2014 7th International Conference on Intelligent Computation Technology and Automation, Changsha, 2014, pp. 268-271.

[15] Luo Xiaoquan, pan Shanliang. Application Research of multisensor data fusion in fire detection [J]. Wireless communication technology, 2019, 28 (03): 57-62

\section{Biography}

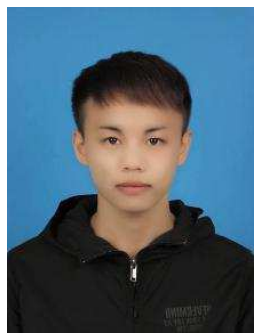

Huang Ye was born in Xichang City, Sichuan Province, China in 1993. He received a bachelor's degree from Sichuan University of Science and Engineering, and now he is a graduate student in the school of Automation and Information Engineering of Sichuan University of Science and Engineering. His main research direction is intelligent control and system optimization.

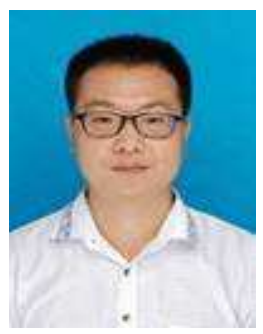

Wang Xiaogang was born in Baoji City, Shanxi Province, China in 1984. He received his $\mathrm{Ph}$. D degree from the Chongqing University, Chongqing, China. He is currently an associate professor, with Artificial Intelligence Key Laboratory of Sichuan Province, School of Automation and Information Engineering, Sichuan University of Science and Engineering. His current interests are in the area of wireless sensor network and security, IoT, artificial intelligence.

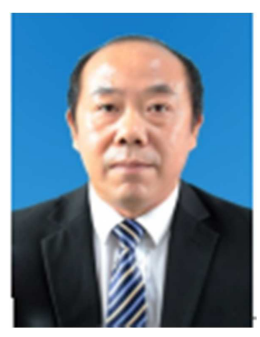

Gan Shuchuan was born in 1969. He obtained his bachelor's degree from North China Electric Power University in 1991. He is currently an associate professor school of Automation and Information Engineering of Sichuan University of Science and Engineering. He is currently mainly engaged in industrial fieldbus application technology, intelligent control, smart grid and other aspects of scientific research. 